\title{
L'enseignement bilingue pour sourds en France vu sous l'angle de la didactique des langues
}

\author{
Educação bilíngüe para surdos na França \\ a partir da perspectiva do ensino de línguas
}

\author{
Véronique GEFFROY \\ Docteur en Didactique des Langues
}

François LE ROUX

Enseignant de/en LSF

Résumé: En France, il existe un dispositif particulier d'enseignement dans lequel toutes les fonctions de l'école sont assurées et qui donne accès aux programmes nationaux officiels grâce à une autre langue que le français : ce sont les classes immersives en langue régionale. La même démarche peut être appliquée à la LSF. En effet, l'accès au français seul suppose une dépense cognitive considérable pour les enfants déficients auditifs, pour leur famille et même pour les enseignants.En faire une condition d'accès à l'écrit détourne le passage par l'école de ses fonctions essentielles : la transmission des connaissances, l'accompagnement métacognitif, la socialisation et la régulation des échanges, diverses activités langagières dont le travail métalinguistique indispensable à la lecture, etc. Dans cet article $^{1}$, nous nous proposons de regarder la scène scolaire avec l'éclairage de la didactique des langues, et de faire en sorte que la rééducation du français ne soit pas le seul projecteur allumé dans la scolarité des enfants sourds.

Mot clés: enseignement bilingue, LSF, classes immersives, oralisme, langue d'enseignement, langue première, sourd, déficience auditive

Resumo: Na França, existe um dispositivo específico de ensino no qual todas as funções da escola são asseguradas e que dá acesso aos programas nacionais oficiais através de uma língua diferente do francês: trata-se das classes imersivas em língua regional. A mesma abordagem pode ser aplicada à LSF. De fato, o acesso somente ao francês supõe um esforço cognitivo considerável para as crianças com deficiência auditiva, para sua família e até mesmo para os professores. Estabelecer essa estratégia como condição de acesso à escrita desvia a passagem pela escola de suas funções essenciais: a transmissão dos conhecimentos, o acompanhamento metacongnitivo, a socialização e as trocas, diversas atividades linguageiras dentre os quais o trabalho metalinguístico indispensável à leitura, etc. Nesse artigo, nos propomos olhar a cena escolar sob a luz da didática das línguas, de forma que a reabilitação do francês não seja o único parâmetro na educação de crianças surdas.

Palavras-Chave: Educação Bilíngue, LSF, Turmas iImersivas, oralismo, primeira língua, surdo, deficiente auditivo

\section{Le monolinguisme à la française}

\subsection{Du monolinguisme en général}

Dans le cadre de programmes scolaires généralistes, il est question que les enfants s'intéressent aux autres langues que la langue nationale dès la classe de CE1. Pendant

\footnotetext{
${ }^{1}$ Article qui reprend et réactualise celui publié par les mêmes auteurs sous l'intitulé « Enseignement bilingue, la perspective ouverte par la didactique des langues », La Nouvelle Revue de l'AIS, hors série n 5 , édition de l'INSHEA, juin 2005, pp. 19-36.
} 
longtemps et jusqu'à récemment, pouvoir s'exprimer dans plusieurs langues et passer d'une langue à une autre étaient des compétences que beaucoup de franco-français maîtrisaient tardivement, voire jamais. De nos jours, s'affirme une volonté forte pour que cela change.

Bien sûr, tout le monde rêve de voir son enfant apprendre l'anglais, à l'âge de 7 ans. Chacun semble convaincu que c'est là un investissement sur l'avenir, parce que toute carrière dans l'informatique, le commerce, le tourisme sera compromise sans l'anglais. On peut trouver sans hésiter quelques bons arguments pour justifier une introduction précoce de l'anglais dès les petites classes, et son maintien tout au long de la scolarité : d'une part viser une connaissance littéraire de cette langue, d'autre part envisager de suivre une partie de sa scolarité en anglais, d'où une volonté de préparer par anticipation un certain confort communicationnel. Et puis, bien connaître plusieurs langues ouvre aussi aux métiers de l'interprétation et de la traduction.

En sortant d'un triste monolinguisme à la française, on découvre la capacité de changer de langue tout en gardant une certaine efficacité communicative. Il n'est heureusement pas nécessaire d'être bilingue pour communiquer dans une autre langue. Pour cela, quelles que soient les langues concernées, il est bon de s'entraîner à déverbaliser pour réexprimer sans transcodage. Quelques bons locuteurs bilingues le font occasionnellement, sans être pour autant des professionnels de l'interprétation, car ces stratégies sont à la portée de tous.

Force est de constater que des enfants ayant appris tôt des langues très éloignées de la nôtre (langues sémitiques, slaves, orientales, gestuelles) ont de vrais atouts pour les langues qui nous sont plus familières.

Finalement, en s'appuyant :

- sur le fourmillement intellectuel et la plasticité cérébrale des enfants,

- sur des jeux de répétitions et d'imitation de formules, de chansons, d'histoires permettant $d$ 'acquérir un système phonologique très différent,

- $\quad$ sur le fait qu'eux osent encore se tromper ou mal prononcer, sans avoir peur d'être ridicule,

- sur leur curiosité pour tout ce qui sort de l'ordinaire,

faire apprendre n'importe quelle langue à nos chères "têtes blondes", en-dehors d'une pratique intra-familiale, devient possible. Les compétences acquises lors de l'acquisition d'une autre langue sont transférables à toute nouvelle expérience linguistique. L'enfant pourrait alors 
apprendre tardivement l'anglais (fonctionnel ici) ou d'autres langues latines par exemple, en fin de collège ou au lycée, en ayant manipulé précocement d'autres langues plus exotiques²

Connaître plusieurs langues permet aussi de développer la capacité à se décentrer, à adopter un autre point de vue, à toucher du doigt qu'il n'y a pas qu'une façon de faire ou de dire : bref, la capacité à relativiser. C'est donc quasiment une initiation à l'esprit critique, dans le bon sens du terme, et pourquoi pas d'une certaine façon, une toute première leçon de philosophie... ce qui justifierait encore plus de s'y intéresser à l'âge de raison !

\subsection{Dans le contexte de l'enseignement aux enfants sourds.}

Puisqu'il est enviable pour tous de connaître plusieurs langues et de pouvoir naviguer d'un univers linguistique à un autre, pourquoi ne pas l'encourager en particulier, chez les enfants sourds? Au nom de l'enrichissement linguistique, présenter deux langues à un enfant bien entendant ou à un enfant sourd en capacité de participer à des discussions en langue vocale ne semble pas choquant. Que cet argument soit négligé d'office s'agissant de la présence d'une langue gestuelle à côté de la langue nationale en construction est par contre bien surprenant. Pourquoi en effet privilégier sciemment (et souvent exclusivement) le français, et retirer aux enfants sourds cette chance de connaître deux langues en ne leur proposant pas une acquisition sérieuse de la LSF ? Pourquoi mettre à ce point l'accent sur la langue nationale (dans sa modalité orale prétextant que cette modalité est indispensable à l'entrée dans l'écrit) au prix de beaucoup d'efforts pour un résultat ingrat? Finalement, le plus souvent, le français reste une langue peu investie par les sourds, malgré le nombre d'heures et d'intervenants qui y ont été consacrés et malgré l'énergie que l'enfant sourd luimême peut y mettre. Il est toujours surprenant de constater que les spécialistes de l'éducation des sourds ne s'emparent pas volontiers de la parole des sourds adultes qui ont une connaissance de la surdité vécue "de l'intérieur". C'est pourtant une posture adoptée dans de multiples autres situations ${ }^{3}$.

\footnotetext{
${ }^{2}$ Ceci inclut d'ailleurs les langues des populations issues de l'immigration vivant en France.

${ }^{3}$ Je ne prendrai ici qu'un seul exemple, mais ils seraient nombreux : le cas des enfants de parents divorcés, situation de plus en plus fréquente. Il est admis que la séparation parentale, même bien menée, a des incidences qui se révèlent des années plus tard. Certains spéciialistes s'appuient sur Françoise Dolto (qui défendit par ailleurs la langue des Signes pour les enfants sourds) et justifie cet état de fait par la "dynamique de l'inconscient". On connaît aussi des ouvrages qui rassemblent des témoignages d'enfants majeurs issus de couples divorcés, même parfois âgés, quant aux difficultés rencontrées. Et tout le monde s'appuie sur cette connaissance expérientielle reconnue des enfants du divorce pour construire et améliorer les décisions concernant les plus jeunes dans le cas de divorces en cours.
} 
Nous vous proposons de regarder rapidement en quoi les Langues signées sont particulières pour mieux comprendre ce qui en découle.

\begin{tabular}{|c|c|}
\hline Langues autres que la LSF & La Langue des Signes \\
\hline $\begin{array}{l}\text { MODALITÉ AUDIO-PHONATOIRE } \\
\text { - Réception par l'oreille (seuls quelques éléments sont } \\
\text { perçus par la vue). Discrimination de caractéristiques } \\
\text { acoustiques. } \\
\text { - Production bucco-pharyngée qui suppose un } \\
\text { « entraînement » long jusqu'à l'obtention d'une } \\
\text { production compréhensible et conforme, travail qu'on } \\
\text { sous-estime souvent lorsqu'on y est parvenu } \\
\text { naturellement! } \\
\text { - Déroulement principalement linéaire, avec quelques } \\
\text { informations simultanées parmi lesquelles : } \\
\text { 1/ différents éléments phonologiques (niveau } \\
\text { moteur de petite échelle). } \\
\text { 2/ accent tonique } \\
\text { 3/ intonation +/- ligne mélodique (textes chantés } \\
\text { par exemple) } \\
\text { 4/ gestes co-verbaux, pouvant aussi être de simples } \\
\text { pointages. } \\
\text { 5/ regard posé principalement sur l'interlocuteur } \\
\text { (dans la culture française). L'utilisation du regard } \\
\text { est codifiée socialement et peut s'apparenter à la } \\
\text { gestualité coverbale ou même accompagner cette } \\
\text { dernière. }\end{array}$ & $\begin{array}{l}\text { MODALITÉ VISUO-GESTUELLE } \\
\text { - Réception par la vue car la perception sonore des } \\
\text { Sourds est souvent insuffisante pour discriminer la } \\
\text { parole. D'où un appui sur la pensée visuelle et sur les } \\
\text { indices saillants. } \\
\text { - Production corporelle structurée s'appuyant entre } \\
\text { autres choses sur le détournement de la gestualité } \\
\text { naturelle, sur l'imitation de postures et d'attitudes. } \\
\text { - Traitement d'informations simultanées en-dehors du } \\
\text { déroulement linéaire de l'énoncé dans le temps : } \\
\text { 1/ « formants » du signe situés dans la partie } \\
\text { supérieure du corps (un niveau moteur de grande } \\
\text { échelle). } \\
\text { 2/ tonicité des Signes, accélération et ralenti. } \\
\text { 3/ expression faciale, ampleur des Signes } \\
\text { 4/ distinction non discrète entre gestualité co- } \\
\text { verbale et Signes lexicalisés. Les pointages ne sont } \\
\text { plus restreints à la situation. } \\
\text { 5/ utilisation grammaticale ou lexicale du regard et } \\
\text { des positions de tête/épaule/buste. }\end{array}$ \\
\hline $\begin{array}{l}\text { Dans l'apprentissage des langues vocales, certaines } \\
\text { méthodes prennent en compte les aspects gestuels, } \\
\text { depuis peu. }\end{array}$ & $\begin{array}{l}\text { Ces différents points sont pris en compte dans } \\
\text { l'acquisition d'une langue signée. }\end{array}$ \\
\hline
\end{tabular}

Tableau comparatif 1: La question de la modalité utilisée selon la langue

La scolarisation à dominante oraliste suppose un long travail de l'enfant sourd et de son entourage duquel découle une prise de sens dans l'écrit, approximative ou à peine fonctionnelle, et une expression écrite maladroite avec des erreurs dans l'usage du lexique et des tournures morphosyntaxiques que les Français bien-entendants excusent mal de la part de natifs ayant été scolarisés pendant de longues années.

De plus, ce choix élitiste n'offre aucune garantie pour une bonne participation aux situations de communication de la vie courante, comme le montrait déjà B. Mottez, il y a 35 ans ${ }^{5}$.

\footnotetext{
${ }^{4}$ De même que la majuscule est utilisée pour "sourd" lorsqu'il s'agit du choix identitaire ou de l'appartenance à la communauté sourde, elle est utilisée ici pour "signes" lorsqu'il s'agit des unités lexicales d'une langue gestuelle comme la LSF. Chez les adultes en particulier, un positionnement identitaire de Sourd suppose le choix d'une langue des Signes comme langue du quotidien.

${ }^{5}$ Bernard Mottez, La surdité dans la vie de tous les jours, Publication de CTNERHI, 1981. Rapport qui fait suite à une étude effectuée en 1978/1979.
} 
Très exceptionnels sont les Sourds qui ont vraiment pris goût au français. Bref, la « francophonie » est très relative parmi les sourds. Il y en a pourtant qui ont savouré le cours de français, qui peuvent dévorer des livres et atteindre un niveau de langue bien supérieur à une utilisation fonctionnelle. À regarder de près cette frange de la population française que sont les sourds bons lecteurs, la corrélation n'est pas si nette entre le choix de la langue de la scolarité et le niveau atteint dans la langue nationale ${ }^{6}$.

Il ne saurait être question de données quantitatives ici car, si les travaux sur bilinguisme additif ${ }^{7}$ et bilinguisme soustractif ont montré que l'apprentissage d'une deuxième langue ne concurrence pas l'acquisition de la première, ce principe est peu présent dans les projets linguistiques mis en œuvre dans l'éducation des sourds. La tendance oraliste refuse toute valeur statistique aux résultats obtenus en lecture dans les filières dites bilingues car l'échantillon est trop faible. Rien n'est dit par contre quant à la représentativité statistique des enfants sourds non bilingues - d'intelligence normale pour une grande majorité - dont les résultats en lecture sont si catastrophiques que cela indique clairement que la manière de procéder n'est pas performante... Pourtant, rien ne change !

UNE ALTERNATIVE... À UN SEUL CHOIX !

\subsubsection{Pour les parents}

Malgré ce qui figure dans la loi française de $2005^{8}$, il n'y a pas de véritable choix pour les parents d'un enfant signalé déficient auditif. À l'effet «blouse blanche » qui survalorise la parole du médecin, s'ajoute le fait que les spécialistes de la physiologie de l'oreille et de la rééducation de la parole ne sont pas nécessairement spécialistes de la surdité ${ }^{9}$.

\footnotetext{
${ }^{6}$ Quand la LSF est présente dans le cursus scolaire, elle n'est jamais proposée dans des proportions satisfaisantes. Un simple coup d'œil sur les emplois du temps permet de constater le peu d'heures d'enseignement de la LSF que peut suivre chaque enfant et certains n'en ont pas du tout. De plus, le nombre d'heures d'enseignement dans une langue de Signes académique, assuré par des Sourds ou des entendants signants, est aussi portion congrue.

${ }^{7}$ On parle ici d'un bilinguisme dans lequel le prestige n'entre pas en compte. On n'établit aucune échelle de valeur entre les langues, par opposition au bilinguisme soustractif, dans lequel une des langues est dévalorisée ou une des langues est survalorisée.

${ }^{8}$ Loi n ${ }^{\circ}$ 2005-102 du 11 février 2005 pour l'égalité des droits et des chances, la participation et la citoyenneté des personnes handicapées.

${ }^{9}$ Après le choc du diagnostic, les familles trouvent beaucoup plus facilement des relais dans le champ de la déficience ou celui de la réducation (les mots choisis ici ne sont pas anodins) que dans le champ de la sociolinguistique. Et l'effet «blouse blanche » est aussi très prégnant au sein des équipes ayant en charge la scolarisation des déficients auditifs dans les étabalissements spécialisés.
} 
À propos du choix de la langue par les parents, dans l'éducation de leur(s) enfant(s) sourd(s), la loi de 2005 a repris l'article 33 d'une loi de 1991. Un des effets de cette loi plus ancienne sur le choix parental avait conduit les familles à se domicilier à proximité d'une école offrant une éducation bilingue à l'enfant : c'est-à-dire que les parents demandaient une mutation et que toute la famille déménageait. Étonnamment, la loi de février 2005 n'y changera rien, au contraire, car le principe de l'inscription dans l'école proche du domicile y est affirmé dans l'idée de défendre "l'égalité des droits et des chances" pour les enfants en situation de handicap. Pour assurer le droit à leur enfants sourd se suivre une scolarité dans une langue riche et confortable, ce sera donc encore la famille qui devra se rapprocher des rares écoles ou la LSF est la langue d'enseignement. Les familles sont de plus en plus souvent au milieu d'un nouveau conflit entre les "blouses blanches ${ }^{10 "}$ qui se chargent de la rééducation de la parole et les "blouses grises ${ }^{11 " ~ q u i ~ p r o ̂ n e n t ~ l ' i n c l u s i o n ~ s c o l a i r e ~ t r e ̀ s ~ p e u ~ d e ~ L S F, ~ v o i r e ~ p a s ~}$ du tout. Même quand les tâches sont bien réparties et que les deux secteurs professionnels travaillent en bonne entente, la garantie d'un parcours scolaire en LSF n'est souvent pas possible à accorder.

Or, un enfant sourd ne sera jamais entendant même après une rééducation oraliste fructueuse, et quelle que soit l'efficacité du mode de correction auditive mis en place.

La démarche des parents d'enfants sourds scolarisés en classe bilingue entretient des similitudes avec celle des parents ayant fait le choix de classes immersives en langue régionale (que nous décrirons plus loin). Mais on peut noter trois différences importantes concernant :

- La question de la scolarisation des enfants dans une langue régionale ne se pose pas aux parents dans un contexte médicalisé. Elle se pose en termes de patrimoine, même quand certains parents ne sont pas locuteurs de la langue régionale. Or, les parents d'enfants sourds ayant choisi la LSF pour leur enfant ne parviennent pas à faire entendre que leur choix d'une langue gestuelle pour l'enfant s'appuie sur l'idée de patrimoine culturel.

- Beaucoup de détracteurs des classes en langue régionale font remarquer que la démarche des parents est si peu banale qu'elle sous-entend une véritable motivation, qui expliquerait les résultats positifs de ce mode de scolarisation. Le terme d'élitisme est même souvent prononcé. À propos de l'enseignement en LSF, la motivation des parents est aussi très porteuse pour l'enfant, de même que le confort communicationnel. Mais le terme

\footnotetext{
${ }^{10}$ Professionnels du secteur médical et para-médical.

${ }^{11}$ Professionnels du secteur éducatif qui ont parfois appris un peu la LSF, mais ont un niveau trop faible pour intervenir dans la tra,smission des connaissance ou l'accompganement au raisonnement.
} 
d'élitisme ne saurait s'appliquer pour les mêmes raisons. En effet, les témoignages des parents ayant accompagné leur enfant dans un projet oraliste jusqu'aux résultats escomptés présentent davantage d'aspects élitistes ${ }^{12}$.

Ceci dit, le choix de scolariser ses enfants dans une langue régionale est une décision parentale qui découle d'une réflexion et d'une maturation préalable à la mise en œuvre de ce projet particulier. Compte tenu de la rareté des dispositifs bilingues pour sourds, le même raisonnement pourrait s'appliquer aux parents d'un enfant déficient auditif, alors qu'au moment du diagnostic, ils se trouvent face en réalité à un choix entre plusieurs formes de souffrance. Aucune configuration n'en est exempte :

- souffrance de choisir un projet oraliste dont la violence peut n'apparaître que (beaucoup) plus tard, entre autres après la découverte du mode de vie gestuel ${ }^{13}$.

- ou souffrance de devoir s'approprier une langue qui n'est pas la sienne pour son enfant. C'est un vrai renoncement pour les parents qui l'ont fait et il leur faut admettre que leur enfant sera meilleur qu'eux dans les échanges, que la fratrie va s'en trouver bouleversée, que les berceuses et les histoires du soir seront laborieuses... Ce n'est pas chose facile de se dire que la langue première de son enfant sera bien différente de la sienne, qui plus est, une langue stigmatisée dans la société. Le cas de la surdité est unique en cela ${ }^{14}$.

Ainsi, on comprendra que la façon la plus répandue d'éluder la question de la langue entre parent et enfant est de se conformer à ce que toute la société insuffle : l'enfant doit apprendre la langue de la société adulte environnante. Et les parents sont imprégnés tacitement de ce point de vue que tout concourt à promouvoir, au lieu d'être éclairés et accompagnés pour faire un vrai choix. Les parents qui font le choix réfléchi de l'oralisme ne sont pas condamnables. Rien n'est simple et le jeu est brouillé.

\footnotetext{
${ }^{12}$ Entre l'Abbé de l'Épée en France et son détracteur allemand Heinicke, l'argument était déjà très présent au $18^{\mathrm{e}}$ siècle.

${ }^{13}$ Cela peut intervenir lors d'une rencontre avec le milieu sourd ou par la révolte de l'enfant devenu adolescent et revendiquant une communication gestuelle, parce que confortable pour lui. Les cheminements ne sont jamais rectilignes. Mais les adolescents sourds scolarisés en intégration trouvent souvent un groupe d'appartenance au sein de la communauté sourde. L'impression de 'communion' est à portée de main dans cette nouvelle tribu, bien plus que dans la famille ou même auprès des amis d'enfance entendants. L'idée que l'oralisme demandé à l'enfant est une forme de harcèlement viendra alimenter les éventuels conflits familiaux et raviver la souffrance parentale qui a présidé au choix de la langue fait pour l'enfant, alors petit. Un jeu assez similaire peut aussi intervenir entre enseignants non signants et élève sourd.

${ }^{14}$ Même dans le cas d'une adoption tardive, par exemple, un parent peut s'intéresser énormément à la langue d'origine de l'enfant. La question ne se pose pas dans les mêmes termes que pour l'enfant sourd, bien qu'on considère souvent à juste titre la question sous l'angle socio-lingusitique.
} 


\subsubsection{Pour l'enfant.}

Un enfant entendant a d'emblée pour langue première la langue de ses parents ${ }^{15}$. Puis il rencontrera d'autres langues au cours de sa scolarité, qu'il abordera avec la compétence de locuteur qu'il s'est construite dans sa(ses) langue(s) maternelle(s).

On constate que, en l'absence d'enseignement de la LSF, les enfants sourds ont tellement besoin de communiquer qu'ils sont capables de créer des lectes gestuels, pendant les récréations, les repas, la vie à l'internat ou avec une des membres de leur famille qui se montre réceptif. Les processus de lexicalisation et les structures de leurs discours sont proches de ceux qui ont été identifiés chez les Sourds ayant appris une langue dans des institutions avec une histoire longue ${ }^{16}$.

Par ailleurs, l'école est présentée comme un lieu privilégié de socialisation, dès le plus jeune âge. Pourtant, dans le cas de la participation scolaire sans une vraie communication, la socialisation est compromise ${ }^{17}$.

Dans les programmes officiels, et notamment dans ceux qui s'attachent à la scolarisation des tout-petits ", la communication et le langage, ou l'affectivité et les relations sociales, sont les points d'ancrage du travail. Au premier abord, on pourrait penser que ces aspects sont entravés par la surdité, ou plus exactement par le déficit communicationnel qui en découle. Néanmoins, si l'adulte s'inscrit dans une communication visuelle accessible à l'enfant sourd et accueille les propositions de ce dernier, l'échange n'est plus marqué par le handicap. Les différents domaines d'activité pour structurer le langage peuvent alors s'effectuer au même titre que pour un public d'enfants entendants à l'école maternelle, c'està-dire : «le langage au cœur des apprentissages », «vivre ensemble », « agir et s'exprimer avec son corps », « découvrir le monde », et « la sensibilité, l'imagination et la création ».

Il n'y est fait aucune allusion à une symbolique gestuelle qui n'est pas l'apanage des seuls sourds, ni bien sûr à l'iconicité des langues gestuelles. L'usage de symboles gestuels,

\footnotetext{
${ }^{15}$ Il peut rencontrer précocement deux langues ou plus, configuration qu'un pays traditionnellement monolingue comme la France voit comme une complication, alors qu'elle devrait être banale sur cette "terre d'accueil". Quand un enfant entendant a des parents Sourds, sa langue maternelle est la LSF.

16 FUSELLIER-SOUZA I. (2001) « La création gestuelle des individus sourds isolés. De l'édification conceptuelle et linguistique à la sémiogénèse des langues des signes. » In AILE (Acquisition et Interaction en Langue Etrangère) $\mathrm{n}^{\circ} 15$, Paris. pp. 61-96.

${ }^{17}$ Malheureusement, la socialisation est aussi compromise dans la plupart des institutions pour enfants sourds qui privilégient l'acquisition du français plutôt que la socialisation et l'acquisition des connaissances. Or, c'est de loin le cas le plus fréquent, même s'il est inavoué ! Il faudrait mettre à plat les distorsions entre projets institutionnels et éléments de terrain tangibles.
} 
accessible à tous ${ }^{18}$, est pourtant un formidable procédé de symbolisation de l'expérience perceptivo-pratique, comme le montrent les travaux de Christian Cuxac ${ }^{19}$ et d'Ivani FusellierSouza $^{20}$.L'institution scolaire en France est dans une forme de déni.

Avant de passer aux incidences de la surdité sur l'acte d'enseigner, voyons comment se pose la question de l'acquisition d'une nouvelle langue pour le public d'âge scolaire.

\begin{tabular}{|c|c|}
\hline Langues autres que la LSF & La Langue des Signes \\
\hline $\begin{array}{l}\text { - Les élèves apprennent des langues vivantes proposées } \\
\text { dans les programmes scolaires. Autant dire que, le plus } \\
\text { souvent, quelqu'un d'autre que l'enfant a décidé qu'il } \\
\text { lui faudrait apprendre une autre langue. } \\
\text { 1) En général, trois heures hebdomadaires sont } \\
\text { prévues, ou davantage dans les options « langue } \\
\text { renforcée ». } \\
\text { Avec les classes « européennes », certaines } \\
\text { disciplines (scientifiques, souvent) sont dispensées } \\
\text { dans une autre langue que le français. } \\
\text { 2) Quelques déficients auditifs oralisés ou signants } \\
\text { répondent à l'obligation d'apprendre une langue } \\
\text { vivante dans leur cursus. Cela nécessite des } \\
\text { adaptations que nous ne détaillerons pas ici. } \\
\text { - Il y a parfois des dérives : certaines familles font le } \\
\text { choix pour leur enfant d'une langue vivante rare dans le } \\
\text { but de l'inscrire dans un établissement ayant une bonne } \\
\text { réputation. De plus en plus souvent, lorsque des élèves } \\
\text { veulent suivre l'enseignement d'une langue qui n'existe } \\
\text { pas dans leur établissement, il y a des aménagements } \\
\text { entre établissements pour les heures de cours et les } \\
\text { élèves se déplacent temporairement (ceci est vrai pour } \\
\text { les grandes villes, et à Paris notamment, où l'offre est } \\
\text { très variée). }\end{array}$ & $\begin{array}{l}\text { - Concernant les enfants déficients auditifs, peu } \\
\text { d'établissements refusent ouvertement la LSF de nos } \\
\text { jours. Mais, en matière d'acquisition guidée de la LSF, } \\
\text { il y a rarement trois heures hebdomadaires de cours de } \\
\text { LSF comme pour les langues vivantes. } \\
\text { Malgré la parution de programmes officiels pour } \\
\text { l'enseignement de la LSF en } 2008 \text { et } 2209 \text {, il est } \\
\text { difficile de généraliser ou de mesurer à quelle échelle } \\
\text { un enseignement disciplinaire en LSF est proposé. } \\
\text { On assiste souvent à un saupoudrage de la LSF dans } \\
\text { l'emploi du temps, sans commune mesure avec la } \\
\text { présence renforcée du français dès le plus jeune âge } \\
\\
\text { - Dans de rares cas, la LSF est la langue } \\
\text { d'enseignement et permet en particulier d'observer les } \\
\text { structures du français et de faire des analyses de textes } \\
\text { littéraires. Les résultats sont intéressants, mais il } \\
\text { faudrait un élargissement important de tels projets pour } \\
\text { arrêter de les considérer comme expérimentaux. }\end{array}$ \\
\hline
\end{tabular}

Tableau comparatif 2 : Apprendre une nouvelle langue pour un public d'âge scolaire

\footnotetext{
18 Nous avons enfoui ce procédé en grandissant et sommes en grande difficulté quand il faut y revenir au moment d'apprendre la LSF. Peu de recherches porte sur cette question chez l'adulte, en-dehors de la thèse de Françoise Morillon.

${ }^{19}$ CUXAC Christian. «Apprendre la LSF ». In : PELLION François (dir.). Surdité et souffrance psychique. Paris : Ellipses, 2001, p. 33-38.

20 FUSELLIER-SOUZA I. (2001) « La création gestuelle des individus sourds isolés. De l'édification conceptuelle et linguistique à la sémiogénèse des langues des signes. » In AILE (Acquisition et Interaction en Langue Etrangère) n ${ }^{\circ} 15$, Paris. pp. 61-96.

${ }^{21}$ Cela conduit à un pseudo-bilinguisme. Bernard Mottez privilégie le terme de diglossie pour expliquer l'éventuel continuum entre français, français signé et LSF.
} 
Les arrangements proposés pour qu'un élève entendant apprenne la langue de son choix malgré les obstacles font apparaître, par contraste, les procédés dissuasifs que rencontrent les enfants sourds et leur famille pour accéder véritablement à la LSF. Toutefois, sans même entrer dans le détail du degré de déficience auditive, on ne peut pas ignorer les difficultés d'apprentissage et de communication que rencontrent les enfants sourds s'ils sont confinés dans une langue audio-vocale. Heureusement, du point de vue cognitif, les recherches de Cyril Courtin ${ }^{22}$ montrent que les concepts se mettent en place plus tardivement chez les enfants sourds, mais avec les mêmes caractéristiques. N'est-il pas regrettable de se dire par dépit que c'est réparable et qu'il n'est jamais trop tard? Ne vaudrait-il pas mieux de se donner d'emblée des moyens linguistiques efficaces?

Les enfants sourds de parents sourds signants ${ }^{23}$ (ou de façon un peu différente, les enfants qui ont un «aîné » sourd signant dans la famille) ne connaissent pas ces difficultés : ils rencontrent une langue authentique qui permet en particulier que s'installe un discours intérieur, élément très important pour la conceptualisation. Plus qu'une communication visuelle, il faut une langue première, même si ce n'est pas la langue parentale. Sinon les enfants sourds sont les seuls petits d'Homme sous-alimentés dans la compréhension du monde et dans leurs relations, du fait de choix d'adultes. La question qui se pose est donc bien celle du respect de l'enfant, plutôt que du respect d'une différence ou d'une communauté particulière.

\subsubsection{Pour les enseignants}

Nous tous, avec ou sans la loi de février 2005, condamnerions quiconque provoquerait une situation de handicap. Que dire alors de la « situation de handicap partagé » dans laquelle l'enseignant est placé sciemment quand on exige de lui qu'il enseigne sans pouvoir communiquer suffisamment avec son élève.

Cette loi, pensant contribuer au respect de la différence, a établi en réalité un paradoxe : appliquer le même traitement scolaire à toutes les différences et donc, en quelque sorte, renier la différence au lieu de la regarder et de la respecter, et de jouer le jeu de la différentiation. Sans entrer dans le détail de cette loi, qui présente par ailleurs des atouts, remarquons que chacun peut maintenant, de droit, être accueilli dans l'école du périmètre scolaire duquel il dépend.

\footnotetext{
${ }^{22}$ COURTIN C., Actes des colloques «Conceptualisation et surdité » de Suresnes, en particulier celui de mai 2001, La nouvelle revue de l'AIS $\mathrm{n}^{\circ} 17,1^{\mathrm{er}}$ trim 2002, pp. 181-195

${ }^{23}$ «Signant » suppose ici une vraie pratique de la LSF, ce qui ne va pas de soi, et non un bricolage linguistique gestuel.
} 
La surdité, handicap «invisible», devrait essuyer moins de refus que d'autres handicaps : un enfant sourd marche, court, va aux toilettes, monte les étages comme tous les autres enfants. Pas besoin de construire un plan incliné, pas besoin de le porter pendant ses déplacements, pas de machine à écrire particulière, pas de table ni de lampes spéciales... Mais quand on croit prendre en compte la différence de l'enfant sourd en proposant un micro à l'enseignant, en installant éventuellement une boucle magnétique, c'est oublier qu'envoyer un enfant à l'école suppose qu'il y soit instruit et donc qu'il y ait une vraie communication (ou du moins une compréhension satisfaisante pour le « récepteur » qu'est l'élève, à supposer que la frustration de devoir différer ses productions soit tolérable).

Conscients de cet épineux problème, les partisans de l'intégration ont longtemps joué sur l'aménagement de l'emploi du temps et retiraient l'enfant de la classe pour reprendre les contenus avec lui : pourquoi insister pour que l'enfant aille en classe et l'en décrocher aussi souvent, plutôt que d'assurer les contenus dans une langue confortable et l'inciter à rejoindre un groupe d'enfants de son âge sur d'autres situations?

La loi de 2005 incite à mettre en place des moyens de compensation qui, dans le cas des enfants sourds, sont principalement des assistants présents aux côtés de l'enfant, pendant les cours. Il est difficile de savoir ce qu'apportent ces dispositifs lourds en ressources humaines car si l'on met souvent sur le compte de la surdité (ou de l'enfant sourd lui-même) les incompréhensions, il n'y a pas d'outils pour savoir si la réussite d'un élève sourd lui appartient ou revient à une aide humaine. Souvent on reconduit des aides sans avoir évalué en quoi elles ont été fructueuses. La part qui revient à l'enfant et à ses efforts de suppléance est négligée.

Un discours de transmission de connaissances doit s'effectuer dans une langue accessible et confortable pour tous les protagonistes, en particulier pour celui auquel tout est destiné. Mais ici également, se pose la question du respect de l'enseignant qui, si la communication est minimale, ne trouve pas sa place dans la relation avec son public d'apprenants et ne peut pas accomplir la mission qui lui a été confiée. Contrairement à ce qu'on pourrait imaginer, cette remarque ne s'applique pas seulement au cas de l'enseignant qui accueille un enfant sourd en classe ordinaire, mais également à l'immense majorité des enseignants en établissements spécialisés.

L'impossibilité pour l'enseignant d'avoir une communication satisfaisante, provoque un retard dans la scolarité : avant d'être les limites éventuelles de l'enfant, ce sont les limites communicationnelles de l'enseignant qui font qu'une leçon durera plus longtemps ou sera moins approfondie. Devenus adultes, les Sourds n'auront pas pu compenser un tel retard qui 
ne leur est pourtant pas imputable et qui s'est accumulé d'année en année. Au contraire, la société leur fait payer leur faible niveau scolaire : cela pourrait être comparable à une « double peine »!

Les enseignants qui se rendent compte de l'impossibilité dans laquelle le système les place sans les former à mieux communiquer vivent un malaise immense. On pourrait presque comprendre qu'ils éludent la question en défendant le point de vue oraliste ! Quelques-uns vont, eux, jusqu'à quitter les écoles pour sourds, en quelque sorte par honnêteté intellectuelle. La comparaison est difficile ici avec les autres enseignants de langues, pas même avec les enseignants de classe immersives en langue régionale : le contexte est trop spécifique.

Les parents sourds signants connaissant bien cette facette du problème, il semble moins paradoxal de les voir défendre une scolarité en milieu ordinaire pour leurs propres enfants sourds : «Ce serait dommage que mon enfant suive avec des Sourds car le niveau est bas alors qu'il peut suivre avec des entendants. Il a le niveau pour comprendre et il aura un plus grand choix de métiers... », entend-on de leur part. Ces parents ne souhaitent pas un enseignement allégé pour cause de difficulté communicationnelle... et ils sont assez bien placés pour savoir que c'est la réalité de beaucoup de dispositifs spécialisés. Ils réclament un enseignement à hauteur de celui qui est dispensé aux enfants bien entendants du même âge. Avec une certaine lucidité, ils préfèrent se focaliser sur la quête d'un assistant de vie scolaire qui connaisse bien la LSF, maintiennent leurs enfants là où les programmes officiels sont respectés et font tout pour l'orientation de l'enfant ne soit pas pré-programmée. C'est la même raison qui conduit parfois des jeunes sourds à poursuivre par correspondance au-delà du collège.

\section{Un projet scolaire calqué sur les classes immersives}

Le principe des classes immersives en langue régionale s'est établi en France entre 1969 et 1977 selon les cas. Il a maintenant été suffisamment mis à l'épreuve pour qu'on s'y attarde. Ce sont des associations qui s'en sont chargées. Ce type d'écoles concerne 5 langues minoritaires en France et, jusqu'à la création de sections européennes dans l'enseignement secondaire, c'étaient les seules classes dans lesquelles la langue de l'école pouvait être une autre langue que le français. La langue régionale est alors définie comme «langue enseignante et langue enseignée $\gg$.

L'immersion en langue régionale veut être précoce (âge souhaité : 2 ans) et vise à reconstituer l'apprentissage naturel. Les procédés cognitifs sont alors les mêmes dans la 
langue régionale que ceux qui sont développés en français. L'accès à l'écrit se fait également en langue régionale.

Les premières associations du «réveil sourd» se sont appuyées sur les résultats obtenus par les militants des langues régionales pour créer les premières classes bilingues pour sourds en 1984. Leur principe sert encore de référence pour définir le bilinguisme sourd $^{24}$.

Pour eux, la place réservée à la LSF dans le cadre des classes bilingues pour enfants sourds est la même que dans le cadre des classes immersives en langue régionale : la LSF est la langue de transmission des connaissances, c'est aussi la langue qui permet en quelque sorte la cogitation et celle qu'on utilise pour décrire le fonctionnement du français écrit ainsi que le contenu de tout type de discours couché sur le papier.

La comparaison s'arrête là car la situation de la LSF est plus socio-historique que socio-politique.

\begin{tabular}{|c|c|}
\hline Langues autres que la LSF & La Langue des Signes \\
\hline $\begin{array}{l}\text { - Sur les décisions concernant la place qu'une société } \\
\text { fait à une langue dans l'éducation, voir « Que sais-je ? } \\
\text { L'approche interculturelle » de Martine Abdallah- } \\
\text { Pretceille, en } 2010 \text {. }\end{array}$ & $\begin{array}{l}\text { - Pour une approche interculturelle concernant les } \\
\text { sourds, voir l'article de F. Bertin et M. Abdallah- } \\
\text { Pretceille, en } 2005 .\end{array}$ \\
\hline $\begin{array}{l}\text { - Toutes les langues vocales (en-dehors de l'espéranto) } \\
\text { sont liées à un territoire et à une communauté, assez } \\
\text { clairement délimités }{ }^{25} \text {. On voit fleurir des positions } \\
\text { plus ou moins protectionnistes allant des plus nuancées } \\
\text { et d'autres plus extrêmes... }\end{array}$ & $\begin{array}{l}\text { - La LSF est une langue liée à une différence } \\
\text { physiologique (classée par la société parmi les } \\
\text { handicaps et non comme une différence culturelle et } \\
\text { linguistique). Avant tout, l'usage d'une langue } \\
\text { gestuelle est une adaptation judicieuse aux limites que } \\
\text { cette différence impose. Pour les Sourds, ce n'est pas } \\
\text { un choix, elle répond à un besoin vital de communiquer } \\
\text { et participe au bien-être de la personne sourde. }\end{array}$ \\
\hline & - Comparaisons possibles \\
\hline & 1/ Certaines classes bilingues pour Sourds sont \\
\hline $\begin{array}{l}\text { 1/ Pour l'alsacien, le basque, le breton, le catalan, le } \\
\text { corse et l'occitan : l'enfant est scolarisé et apprend à } \\
\text { lire dans la langue régionale. Il transfèrera ses } \\
\text { compétences, et en particulier sa compétence de lecteur }\end{array}$ & $\begin{array}{l}\text { comparables aux classes immersives, à condition de } \\
\text { bien définir la réalité baptisée « classe à projet } \\
\text { bilingue ». La lecture et l'entrée dans le français se font } \\
\text { alors concomitamment en LSF. }\end{array}$ \\
\hline & 2/ On peut reconnaître l'existence d'une diaspora \\
\hline $\begin{array}{l}\text { en compte la diaspora et pas uniquement l'implantation } \\
\text { géographique des Juifs en Israël. }\end{array}$ & $\begin{array}{l}\text { - quand on assiste aux retrouvailles entre anciens élèves } \\
\text { d'une même école de sourds, } \\
\text { - dans le cas de rencontres entre sourds venant de pays } \\
\text { différents qui se sentent plus proches qu'avec les } \\
\text { entendants de leur pays d'origine. }\end{array}$ \\
\hline 3/ Pour ce qui est des langues de pays autrefois & 3/ Il semble inenvisageable que des gens nés sur le \\
\hline
\end{tabular}

\footnotetext{
${ }^{24}$ La thèse d'Elise Leroy, en 2010, traitait de ce point.

${ }^{25}$ On connaît une tentative de formation d'une ville sourde aux USA, à laquelle on peut préférer l'idée de ville signante. Dans ce domaine, les idées extrémistes fleurissent comme ailleurs.
} 
colonisés par la France, leur statut se pose souvent en termes de langue officielle/ langue seconde et les relations entre Langue et pouvoir viennent interférer. territoire français, dans des familles francophones, n'aient pas le français comme langue première.

La société impose aux sourds le français oral parce qu'il est parlé par la majorité des parents, et que c'est la langue de l'école (donc de l'éducation et de la transmission des savoirs) et des média, mais aussi parce que c'est la seule langue nationale en France.Vu que la compétence écrite se construit systématiquement sur la compétence orale dans 1 'école ordinaire, un enfant sourd non oralisant se trouve dans une situation de discrimination structurelle.

Tableau comparatif 3 : Situation socio-politique de la LSF

Certes, il ne faut pas que l'acquisition du français soit remise en cause, mais est-il juste que la société française se préoccupe aussi peu de la place qui sera faite à la LSF ? Ce qui est grave surtout, c'est que la sous-information qui découle du choix linguistique est rarement prise en compte, si ce n'est pour accuser la LSF de ghettoïser les Sourds, alors que les recherches, depuis 40 ans, montrent le contraire.

L'application du principe de tout bilinguisme permettrait d'envisager la LSF comme langue première (et/ou langue maternelle chez les enfants de parents sourds) et le français comme langue seconde. C'est donc souvent ce qui est sous-jacent à propos de «vrai » bilinguisme dans le champ de la surdité.

L'immersion en langue régionale prévoit de compenser le déséquilibre avec la langue dominante pour obtenir un bilinguisme suffisamment équilibré ${ }^{26}$ et propose donc une présence intensive voire exclusive de la langue régionale. On peut se risquer à dire que dans le huis-clos d'une classe où la LSF est la langue d'enseignement, la configuration des langues glisse en fait vers un rapport inverse : la LSF étant la langue de prédilection, parce que visuogestuelle, il faudra un renforcement du français (langue dominante, envahissante à l'extérieur de l'école, mais difficilement accessible aux Sourds) pour tendre vers un bilinguisme équilibré.

C'est le grand défi que doivent relever les classes bilingues pour sourds, dans lesquelles les acquisitions autres que le français se font cependant dans un bon confort pédagogique.

\footnotetext{
${ }^{26}$ À titre indicatif, les classes immersives Diwan en breton proposent $2 \mathrm{~h}$ de cours en français à partir du CE. À l'entrée au collège, $22 \mathrm{~h} 30$ d'enseignement s'effectuent en breton et $6 \mathrm{~h} 30$ en français. Dès la classe de 4 ème , intervient une troisième langue d'enseignement, avec 5 h30 de cours en anglais (voir site Diwan).
} 
Dans les classes immersives en langue régionale, il est précisé que les enseignants doivent rectifier les erreurs des élèves dans la langue-cible, sinon la langue de la classe glisse vers un idiolecte ${ }^{27}$. En règle générale, il suffit qu'une personne identifiée, même tacitement, comme référent de la langue régionale reformule correctement et les élèves admettent cette formulation comme modèle. Cela doit être fait sans commentaire dévalorisant. Les corrections entre élèves eux-mêmes ont aussi de la valeur, et elles éveillent la conscience linguistique car elles supposent une observation des mécanismes de la langue, mais on mesure l'importance du choix des "nourrices" linguistiques.

Pour rester dans le parallèle entre la scolarisation d'enfants déficients auditifs, nous distinguerons trois grandes configurations, tout en sachant que la réalité n'est jamais aussi tranchée.

a) Certains élèves dits malentendants peuvent avoir suivi tout ou partie de leur scolarité en intégration. Le niveau atteint en français est très variable, selon les individus et leur parcours. La réussite scolaire n'atteint pas dans cette population des chiffres approchant ceux d'une population entendante ayant des capacités intellectuelles supposées équivalentes ; loin de là. Lorsqu'un enfant rejoint une classe en LSF après un parcours en intégration, le parallèle avec les classes immersives régionales est alors possible, mais c'est une immersion tardive.

b) Plus rares sont les enfants sourds scolarisés en immersion dans la LSF, les moyens d'accéder au français écrit dans ce cadre scolaire sont mis en œuvre après l'entrée dans la LSF. Le français étant la langue officielle, elle doit être enseignée aux Sourds dans l'école pour un meilleur accès au savoir collectif, au moins dans sa modalité écrite. Pour l'enseignement $\mathrm{du}$ français écrit, on s'appuiera sur les compétences linguistiques et communicationnelles développées dans la langue de l'école (la LSF) et non l'inverse. L'accès au français oral pourra être pris en charge en-dehors de l'école. C'est le seul cas dans lequel on puisse parler de bilinguisme additif.

c) Pour la plupart des autres enfants sourds, que ce soit suite à un échec de l'intégration scolaire ou non, les apprentissages se font dans un entre-deux linguistique très dommageable. En effet, aucune des deux langues n'est suffisamment solide; il est alors incongru de parler de bilinguisme. Les enfants comme leurs enseignants sont dans une interlangue étayée par des langues très lacunaires de part et d'autre. Chaque protagoniste

\footnotetext{
${ }^{27}$ Concernant les enfants sourds, cette remarque est intéressante : souvent les «nourrices linguistiques » en LSF, surtout les pairs et les aînés, se contentent de s'assurer de la compréhension du sens et négligent la formulation.
} 
s'appuie sur un lecte d'apprenant qu'il a pourtant besoin d'utiliser comme une langue. Ce n'est jamais le cas dans les autres configurations d'acquisition de langues vocales, que l'entrée dans la nouvelle langue se fasse précocement ou tardivement.

Les enseignants de classes immersives en langue régionales ont remarqué aussi que la réussite du bilinguisme est vraiment meilleure s'il y a investissement des parents ${ }^{28}$. Dans son intervention à l'université d'été de l'association 2LPE en juillet 2004, Felipe Hamel faisait état de recherches de psycholinguistes canadiens : l'immersion précoce donne de meilleurs résultats quant à la langue d'enseignement, mais également pour ce qui concerne la langue dominante hors de l'école, pour les mathématiques, pour le maniement de l'abstraction et du symbolisme, ainsi que pour la résolution de problèmes. Nous ne reviendrons pas ici sur ce qui a été dit dans le paragraphe sur l'enfant : pourvu que la langue d'évaluation ne vienne pas interférer sur l'objet de l'évaluation, il apparaît que l'ensemble des démarches non strictement linguistique est accessible par la LSF. Le raisonnement pourrait même être poussé plus loin : contrairement à ce que préconise le principe de la «méthode directe » pour l'apprentissage d'une langue, enseigner le français en français semble moins probant que d'enseigner le français/ langue-cible, par le truchement d'une autre langue.

Enfin, les écoles immersives préfèrent les classes à plusieurs niveaux, car l'enseignant peut mettre en valeur les possibilités des élèves les plus avancés. Le maniement de la languecible par des enfants pour s'adresser à d'autres enfants a un impact fort sur l'acquisition. Les plus jeunes prennent ainsi conscience du contrat didactique.

Justement, avec le "réveil sourd" des années 1980, on a pu découvrir comment l'enfant Sourd de parents entendants apprend la LS par ses pairs, mais surtout par ses aînés. Pour preuve, parmi les pairs (sourds), les enfants Sourds de parents Sourds ne jouent un rôle que s'ils sont des aînés. Les aînés Sourds de parents Sourds sont des informateurs sur les choses de la vie. L'enfant Sourd s'adresse à un aîné qui sait ou demande à ses parents et qui rapporte ensuite la réponse. Les aînés (de parents sourds ou entendants) sont aussi des informateurs quant aux contenus de $\operatorname{cours}^{29}$.

Depuis la loi de février 2005, l'information est centralisée au sein de «maisons départementales" quel que soit le handicap. Même si ces lieux parviennent à prendre une certaine distance par rapport au milieu médical, même si la LSF y est présentée à sa juste valeur, les dispositifs existants pour répondre à un choix d'éducation bilingue aux familles

Savoir tôt que certains énoncés sont conformes et d'autres pas fait partie de l'apprentissage d'une langue normée, mais cela sert aussi à apprendre que, dans la vie, il y a des choses qu'on fait et d'autres qu'on ne fait pas !

${ }^{28}$ Nous ne reviendrons pas ici sur l'élitisme parental et institutionnel que suppose l'oralisme. 
d'enfant sourd qui le souhaiteraient sont rares (une dizaine en 2014). Ils reposent sur les épaules de quelques acteurs et sont très fragiles. À l'inverse, les lieux de scolarisation utilisant le français comme langue d'enseignement sont nombreux, parfois depuis plus d'un siècle, et relèvent de circuits de financements bien implantés.

Tant que les choses seront laissées ainsi, la situation de la LSF en milieu scolaire restera longtemps anarchique et ne garantira pas un accompagnement lucide du choix qui est fait par des personnes autres que le principal intéressé.

Il est donc urgent que le paysage change et que :

- des enseignants soient recrutés avec un niveau C1 ou C2 en LSF du référentiel européen,

- des Sourds diplômés puissent intégrer l'Éducation Nationale sur des postes dits à "sujétion spéciale" comme il en existe pour d'autres spécificités,

- des enfants puissent s'inscrire dans de véritables classes d'immersion partout en France $^{30}$, ou que les établissements qui proposent des filières bilingues répondent à des critères précis en la matière,

- que la question de l'interprétation scolaire soit posée en termes clairs

- que les dispositifs bilingues soient pérennisés, avec la titularisation de professionnels reconnus aussi pour leur maîtrise de la LSF.

Sinon, il est à craindre que quelques générations soient encore sacrifiées: des générations d'enfants sourds, des générations de professionnels entendants, des générations de professionnels sourds compétents mais non reconnus. Or, ceux qui auront décidé seront redevables, à l'enfant qui aura grandi, des choix qu'ils auront faits.

$\mathrm{Au}$ sujet de la reconnaissance des compétences des Sourds qui veulent enseigner en milieu scolaire précisément, il n'existe qu'une seule formation d'état au sein de l'Éducation nationale : la Licence Professionnelle qui s'est ouverte en septembre 2004, sous l'égide de la formation permanente de l'Université de Paris 8, mais qui suppose des montages financiers spécifiques ou des aménagements lourds pour les gens qui sont sur poste. Un concours de recrutement pour les enseignants de LSF comme langue optionnelle dans les établissements secondaires a vu le jour en 2010, mais les critères sont les mêmes que pour tout recrutement dans l'Éducation nationale et, malgré certains aménagements dans les épreuves, la sélection

\footnotetext{
${ }^{29}$ Le rapport de B. Mottez, en 1981, s'appuyait sur une collecte très importante de témoignages.

${ }^{30}$ Sur le principe des classes immersives de langue régionale, on pourrait même envisager que des enfants entendants, de la même fratrie ou non, suivent leur scolarité en LSF. Actuellement, cela semblerait aberrant, principalement parce que les contenus officiels ne sont pas respectés dans la classe de déficients auditifs.
} 
est très élitiste. Une préparation à ce concours existe depuis peu : elle se fait donc principalement sur les acquisitions obtenues de haute lutte par les lauréats.

De plus, une fois en poste, la moindre participation des professeurs sourds à un stage en IUFM, ou même à une conférence pédagogique, relève du cas par cas. Pourtant, le potentiel est là et les actuels pédagogues sourds rivalisent d'ingéniosité pour compenser l'absence de supports pédagogiques et pour mutualiser leurs pratiques ${ }^{31}$.

\section{Conclusion}

Si le dispositif ordinaire portait ses fruits, il n'y aurait pas de raison de vouloir appliquer à grande échelle un dispositif de scolarisation marginal et qui relève vraiment d'un choix individuel.

Cependant, malgré l'incrustation à des degrés variables de la LSF dans la scolarité des enfants sourds et dans leur vie familiale, il faut s'interroger sur le fait que l'enseignement actuel ne parvient pas à réduire les difficultés d'accès au français écrit, et sur le faible niveau de culture générale de beaucoup de jeunes Sourds. Dans le même temps, il faut écouter ce que les principaux intéressés ont à nous dire : être attentif aux difficultés qu'ils ont vécues de l'intérieur au lieu de projeter une analyse d'observateur en mirador et ne pas se priver d'entendre (en LSF...) aussi ce que les sourds signeurs qui ont fait des études, qui aiment lire, qui fréquentent assidûment le patrimoine culturel, entre autres, ont à nous dire.

Enfin, les projets scolaires ne s'appuyant pas sur la présence authentique de la LSF comme langue d'enseignement s'appuient finalement sur la résilience des enfants sourds (on pourrait également s'attarder sur la résilience parentale et la résilience familiale nécessaire à la réussite de l'enfant déficient auditif). Quelques uns «s'en sortent», au regard des critères scolaires actuellement en vigueur s'appuyant principalement sur le français comme langue d'enseignement et d'évaluation : ceux-là sont vraiment résilients. Sans tomber dans un misérabilisme inverse, ne doit-on pas s'étonner que l'on conçoive un projet d'éducation qui tout entier s'appuie sur la capacité de petits d'hommes à fonctionner dans la difficulté. Les résultats scolaires ne s'obtiennent plus par les brimades, les humiliations... heureusement. Pour que le temps de la vie consacré à l'école porte ses fruits, l'idée d'un confort communicationnel restera-t-elle un tabou dans le champ de la surdité ?

Nos préjugés nous incitent à étiqueter les entendants comme appartenant à une culture « savante » et les Sourds comme relevant d'une culture «populaire », alors que la plupart des

\footnotetext{
${ }^{31}$ Geffroy Véronique, thèse, 2015.
} 
sourds sont confinés dans une sous-instruction du fait de l'offre institutionnelle plutôt que du fait d'un choix que la loi prétend déléguer aux parents. Dans toute rencontre marquée par le sceau de la différence, deux cultures se nourrissent l'une de l'autre. Rien ne s'oppose en principe à la rencontre entre sourds et entendants, rien en théorie, si ce n'est la représentation de l'Autre et le passé relationnel tendu entre ces deux univers. Mais à travers la question de l'accès aux compétences visées par le système scolaire, on peut dire que les sourds sont assignés à la place que les décideurs entendants leur réserve. C'est pourquoi il est urgent de travailler à une perspective plus humaniste et de ne plus se tromper d'enjeu : la priorité doit être donnée à l'éducation et à l'accès à la Connaissance. L'accès à la langue française en fait partie et apporte une contribution majeure pour s'enrichir à travers elle, plutôt que l'inverse.

\section{Reference}

Ouvrages et Travaux de recherche :

ABDALLAH-PRETCEILLE Martine, L'éducation interculturelle, PUF, Coll. «Que saisje ? $n^{\circ} 3487,3 e$ édition 2010

DALLE-NAZEBI Sophie, Chercheurs, Sourds et Langue des Signes. Le travail d'un objet et de repères linguistiques, Thèse de doctorat en sociologie, Université Toulouse 2 - Le Mirail, http://www.lesiris.free.fr/EetR/Sociologie/theseSDN-resume.pdf

2006.

FUSELLIER-SOUZA Ivani, Sémiogenèse des langues des signes : Étude de langues des signes primaires (LSP) pratiquées par des sourds brésiliens. Thèse de doctorat sous la direction de C. Cuxac, Université Paris 8, Saint-Denis, 2004.

GEFFROY V., La formation des pédagogues sourds : une étude exploratoire. Thèse de doctorat en Didactique des langues et des cultures, Université de Paris 8, 2015.

HAMEL Felipe, Intervention lors de l'université d'été de l'association 2LPE, juillet 2004. http://www.diwanbreizh.org

LEROY Elise, Didactique de la langue des signes française, langue 1, dans les structures d'éducation en langue des signes, Attitudes et stratégies pédagogiques de l'enseignant sourd, Thèse de doctorat en Sciences du langage, Université Paris 8 Saint-Denis Vincennes, 2010.

MOTTEZ Bernard, La surdité dans la vie de tous les jours, Publication de CTNERHI, 1981 (suite à une étude effectuée en 1978/1979)

MORILLON Françoise, Le corps pour le dire, Thèse en didactique des langues, Université de Nantes, Editions ANRT, 2001.

MOTTEZ Bernard, Les sourds existent-ils ? Textes réunis par A. Benvenuto, L'Harmattan, Paris, 2006. 
$\underline{\text { Revues : }}$

CUXAC C., «Apprendre la LSF ». In : PELLION François (dir.). Surdité et souffrance psychique. Paris Ellipses, 2001, p. 33-38.

COURTIN C., Incidence du mode de communication sur les interactions langagières parent entendant-enfant sourd, In Revue SURDITÉS N 3, décembre 2000.

COURTIN C., Actes des colloques «Conceptualisation et surdité » de Suresnes, en particulier celui de mai 2001, La nouvelle revue de l'AIS $n^{\circ} 17,1^{\mathrm{er}}$ trim 2002, pp. 181-195

COURTIN C., LIMOUSIN F., MORGENSTERN A., « Evaluer les compétences linguistiques des enfants en langue des signes, une expérience pionnière » in Language, Interaction and acquisition, John Benjamin Publishing Company, 2010. Disponible sur http://www.benjamins.com/jbp/series/LIA/1-1/art/08cou.pdf

FUSELLIER-SOUZA I., " La création gestuelle des individus sourds isolés. De l'édification conceptuelle et linguistique à la sémiogénèse des langues des signes. » In AILE (Acquisition et Interaction en Langue Etrangère) $\mathrm{n}^{\circ} 15$, Paris, 2001. pp. 61-96.

GEFFROY V., LE ROUX F., «Enseignement bilingue, la perspective ouverte par la didactique des langues », La Nouvelle Revue de l'AIS, hors série n ${ }^{\circ}$, juin 2005, pp. 19-36

GROSJEAN F., «Le droit de l'enfant sourd à grandir bilingue », In Revue SURDITÉS N 3, décembre 2000.

N.R.A.S. $N^{\circ} 23$ : «Enjeux culturels et pédagogiques », revue éditée par l'INS-HEA de Suresnes, $3^{\text {ème }} \quad$ trim. 2003.

En particulier

- Jacob S., L'acquisition de langage par l'enfant sourd p. 31

- Morillon F., Pour la construction d'une discipline, la didactique de la LSF, p. 73

- Bertin F. et Abdallah-Pretceille M., Les enjeux d'une éducation bilingue, p. 91

- Benoît H., Langue des Signes Française, quels enjeux pédagogiques ? p. 113

Surdité et souffrance psychique. Coordonné par PELLION François (dir.), Paris Ellipses, 2001. En particulier les chapitre 3 (Michel Pogol) et chapitre 4 (Christian Cuxac).

Bulletin Officiel de l'Education nationale $n^{\circ} 5$ du 3 février 2005, p. 217 à 223, «Enseignement élémentaire et secondaire, éducation artistique et culturelle. Orientation sur la politique. » 